\title{
Subjective Cognitive Decline in the Community Is Affected at Multiple Aspects of Mental Health and Life Quality: A Cross-Sectional Study of the Community Medicine of Keelung Chang Gung Memorial Hospital
}

\author{
Yi-Chia Wei ${ }^{a-c}$ Li-Yuan Huang ${ }^{c-e}$ Chih-Ken Chen ${ }^{c, f} \quad$ Chemin Lin $^{c, f}$ \\ Yu-Chiau Shyu c Yao-Liang Chenc,g Wen-Yi Huang ${ }^{a}$ Ching-Po Lin ${ }^{b}$ \\ a Department of Neurology, Keelung Chang Gung Memorial Hospital and Chang Gung University \\ College of Medicine, Keelung, Taiwan, ROC; ${ }^{b}$ Institute of Neuroscience, National Yang-Ming \\ University, Taipei, Taiwan, ROC; ${ }^{c}$ Community Medicine Research Center, Keelung Chang Gung \\ Memorial Hospital and Chang Gung University College of Medicine, Keelung, Taiwan, ROC; d Graduate \\ Institute of Clinical Medical Sciences, Chang Gung University, Taoyuan, Taiwan, ROC; ${ }^{\text {eDepartment of }}$ \\ Nursing, Keelung Chang Gung Memorial Hospital, Keelung, Taiwan, ROC; ${ }^{f}$ Department of Psychiatry, \\ Keelung Chang Gung Memorial Hospital and Chang Gung University College of Medicine, Keelung, \\ Taiwan, ROC; ${ }^{9}$ Department of Radiology, Keelung Chang Gung Memorial Hospital and Chang Gung \\ University College of Medicine, Keelung, Taiwan, ROC
}

Keywords

Aging · Cognitive impairment · Subjective cognitive decline · Depression · Quality of life

\begin{abstract}
Background: Subjective cognitive decline (SCD) is one of the early warning signs of objective cognition impairment and dementia. Methods: This cross-sectional study screened SCD and studied multiple domains of mental health, lifestyle, and life quality of the community-dwelling people in the northern coastal region of Taiwan. Results: Among 426 valid AD8 questionnaires, a cutoff of score 2 divided subjects into 115 with SCD (SCD+) and 311 without SCD (SCD-). Analysis of age, sex, body compositions, and blood tests revealed an older age (60.7 \pm 10.9 years) of the $S C D+$ group than the SCD-group ( $57.6 \pm 12.0$ years, $p<0.05)$. Further exclusion of subjects younger than 50 years eliminated age differences and left 100 with $S C D\left(S C D+\_50\right)$ and 229 without (SCD__50). Multidomain comparisons of the SCD+_50 group over the SCD__50 group were made: the Taiwanese Depression Questionnaire found a higher likelihood of depression; the Pittsburgh Sleep Quality Index revealed suboptimal sleep quality; the SF-36 showed inferior function of all 8 aspects of quality of life; the Health-Promoting Lifestyle Profile documented a less health-seeking lifestyle of nutrition, self-actualization, and stress management. Conclusions: Aging increased the SCD risk. People with SCD had suboptimal performance in mental health and life quality in addition to subjective cognition problems.




\section{Introduction}

The incidence of cognitive impairment gradually increases with age, considerably affecting families and health care systems in an aging society. Patients with dementia are those with impairment in activities of daily living caused by cognitive decline. Dementia has affected 47,500,000 people worldwide and is increasing at the rate of 7,700,000 new cases per year [1]. Mild cognitive impairment (MCI) is a cognitive disorder without impairment in activities of daily living [2]; however, most MCI gradually progresses to dementia [3]. Subjective cognitive decline (SCD) is a warning sign of MCI. Eight primary care centers in Galicia, an autonomous community of Spain, jointly conducted a study on patients aged over 50 years with subjective memory complaints, and objective cognitive tests revealed that $46 \%$ of them had cognitive impairment problems [4]. Subjective memory decline or inferior subjective cognitive function can gradually progress over the course of a couple of years to MCI or dementia, which in turn affects personal lives and familial function [5, 6]. Another systematic literature review explored the emotional problems of people aged 50 years with SCD. Integration of cross-sectional and longitudinal study indicated that depression and subjective cognitive problems mutually affect each other and can lead to comorbidity. Anxiety can result from depression over cognitive function loss and is usually alleviated after receiving professional assistance [7]. Therefore, early detection and treatment can reduce the effects of subjective cognitive problems.

The Community Medicine Research Center of Chang Gung Memorial Hospital, Keelung, has conducted a health care program in the northern coastal region of Taiwan (i.e., Anle District in Keelung City; Ruifang, Wanli, and Gongliao Districts in New Taipei City) since 2013. Geriatric dementia care, early screening, early treatment, and holistic care are the main focuses of dementia care in community medicine. Targeting residents of these areas with cognitive impairment, we conducted mental health screenings on participants through questionnaires and provided them with complete neuropsychological assessments and brain imaging. This cross-sectional study aimed to determine the quality of life as well as physiological and psychological conditions of individuals with SCD to improve the application of holistic care to manage the physical and mental health of patients with cognitive impairment.

\section{Materials and Methods}

\section{Community Survey}

The AD8 (dementia screening interview) was used for screening for SCD on adults aged above 30 years in the selected communities. Developed by the University of Washington, the AD8 is a scale comprising 8 descriptive items for the rapid screening of dementia [8]. The questionnaire may be administered to either the participants or their family members. The AD8 is a highly reliable screening tool capable of efficiently distinguishing between individuals who are and are not prone to dementia [9] by inquiring about conditions that may involve cognitive impairment in their daily life. The Chinese version of the AD8 has been validated to identify patients with MCI effectively [10]. For data collection, we inquired whether the participants had experienced the following changes in the past 5 years: (1) problems with judgment; (2) reduced interest in activities and hobbies; (3) repetition of the same questions, stories, or statements; (4) trouble learning how to use a tool, appliance, or gadget; (5) forgetting the month and year; (6) difficulties in handling complicated finances; (7) trouble remembering appointments; and (8) daily problems with thinking and/or memory.

In this one-on-one interview, participants also completed the Taiwanese Depression Questionnaire (TDQ) for assessment of depressive tendencies [11], the Chinese version of the Pitts- 
Fig. 1. Flow chart of the study design. SCD, subjective cognitive decline; TDQ, Taiwanese Depression Questionnaire; PSQI, Pittsburgh Sleep Quality Index; SF-36, the Short-Form (36-item) Health Survey; HPLP-S, short form of the Chinese Health-Promoting Lifestyle Profile.

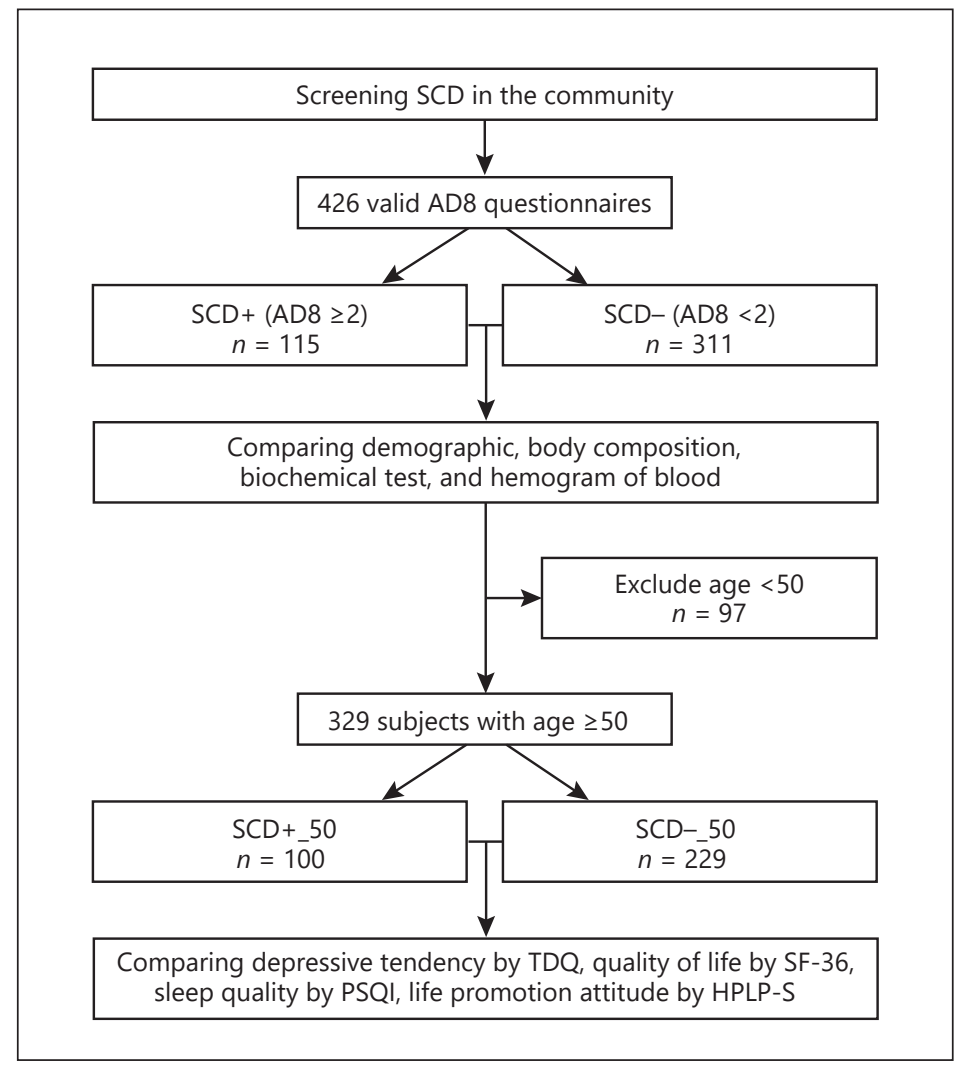

burgh Sleep Quality Index (PSQI) for evaluation of sleep quality [12, 13], the Taiwanese version of the 36-item Short-Form Health Survey (SF-36) for measuring quality of life [14, 15], and the Chinese version short form of the Health-Promoting Lifestyle Profile (HPLP-S) measuring the health-promoting lifestyle [16]. Applying the aforementioned multifaceted assessment instruments, this study gained a comprehensive understanding of the physical and mental health of community-dwelling adults. Protocols of this study have been approved by the institutional review board of the Chang Gung Medical Foundation (IRB No. 201600580B0). All the subjects had been well informed about the study and had given their consents before entering the study.

\section{Statistical Analysis}

The scores obtained from the AD8 questionnaire were subjective measures of participants' cognitive impairment. Cutoff scores distinguished participants with SCD from those without. Items in the AD8 questionnaire were scored 1 point for a "yes" response. Participants who scored 2 points or higher were deemed to have SCD and assigned to the SCD+ group, whereas those receiving zero or 1 point were classified as without SCD and assigned to the SCD - group. Physiological test data (i.e., age, sex, body composition, and blood and biochemical analysis) of both groups were compared to determine the basic physiological differences between those in SCD+ and SCD-. Participants under the age of 50 were further excluded from the study to avoid the potential variations in lifestyle and mental and physical health caused by excessively disparate ages (Fig. 1). TDQ, PSQI, SF-36, and HPLP-S scores of participants older than 50 years with SCD (SCD+_50) were compared with those of participants without (SCD-_50). SPSS 20 statistical software was employed for statistical analyses, namely $\chi^{2}$ testing for categorical data and independent-samples $t$ testing for continuous variables. Additionally, $p<0.05$ was defined as statistical significance. 
Fig. 2. Frequencies of AD8 scores.

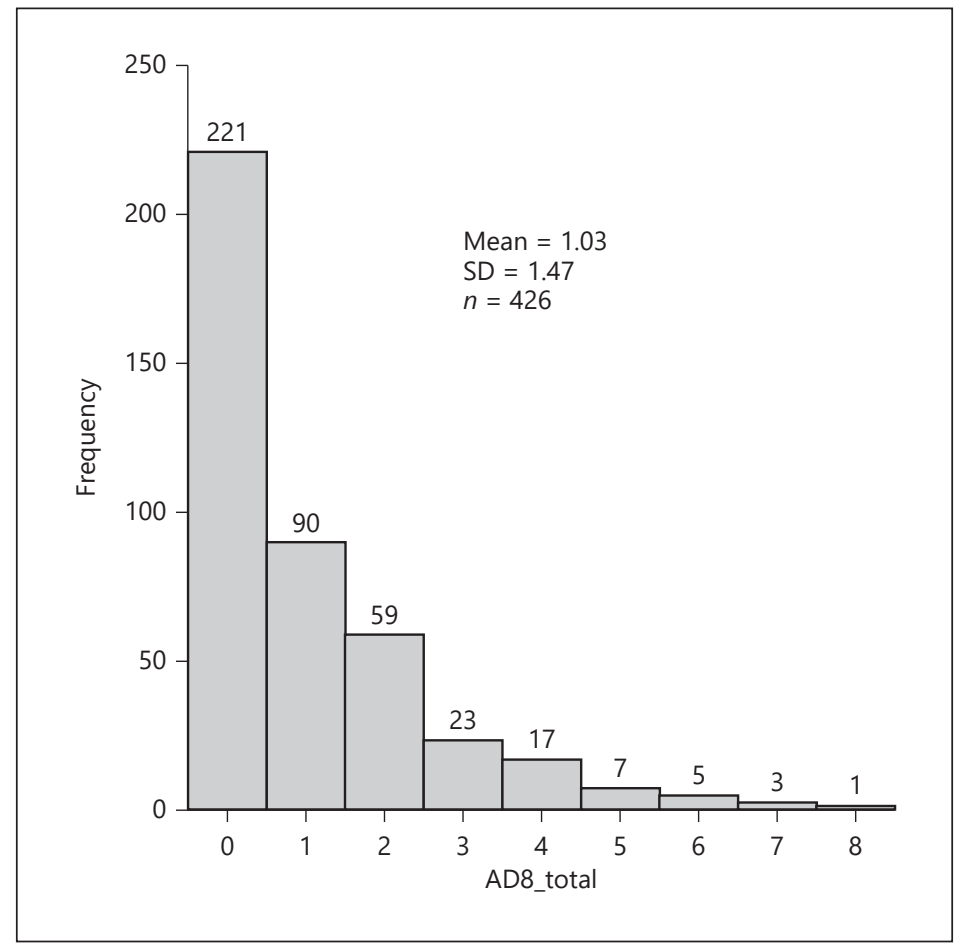

\section{Results}

A total of 426 valid questionnaires were collected from October 2017 through February 2018. The AD8 questionnaire responses were visualized in a histogram (Fig. 2), showing that the majority of participants did not have SCD. A total of 221 participants $(51.9 \%)$ claimed that they had not experienced changes caused by subjective cognitive problems (AD8 $=0$ ), and 90 participants (21.1\%) claimed to have experienced one cognitive problem (AD8 =1). As shown in Figure 2, the 311 participants who had received a score lower than 2 were deemed not to have SCD and thus assigned to the SCD - group. The remaining 115 participants who received a score of 2 or higher were identified as having SCD and thus assigned to the SCD+ group. Regarding group composition, the average age of SCD+ members (60.7 \pm 10.9$)$ was higher than that of SCD - members $(57.6 \pm 12.0)$, with $p<0.05$. The age distribution histogram indicates that ages in both groups exhibited an approximately normal distribution, but SCDmembers were younger (Fig. 3). The average heart rate was the other statistically different criterium between the two groups, with SCD+ members exhibiting an average heart rate of $83.2 \pm 10.5$ beats $/ \mathrm{min}$, whereas that of SCD - was $80.2 \pm 11.8$ beats $/ \mathrm{min}(p<0.05)$. Table 1 demonstrates that the remaining data, namely sex, body composition (i.e., height, weight, and body mass index), blood pressure, and blood test data (i.e., kidney function, liver function index, blood glucose, cholesterol, and complete blood cell count) of the groups exhibited no statistical difference.

Past SCD research conducted in a memory clinic set 50 years as the age threshold [4] to eliminate potential variation caused by age differences. Therefore, in the present study, participants aged younger than 50 years were excluded from subsequent analyses (Fig. 1), resulting in 100 members in the SCD+_50 group and 229 in the SCD-_50 group. The average ages of the two groups thus displayed no statistical difference (SCD+_50: $63.8 \pm 7.6$ years; SCD-_50: $63.3 \pm 7.9$ years), with $p=0.571$. Data of sex, body composition, and blood test indi- 
Fig. 3. Age distribution between SCD+ and SCD- groups. The age distributions are both near normal distribution in SCD- (upper row) and SCD+ (lower row). The curves of normal distributions are shown in each figure.

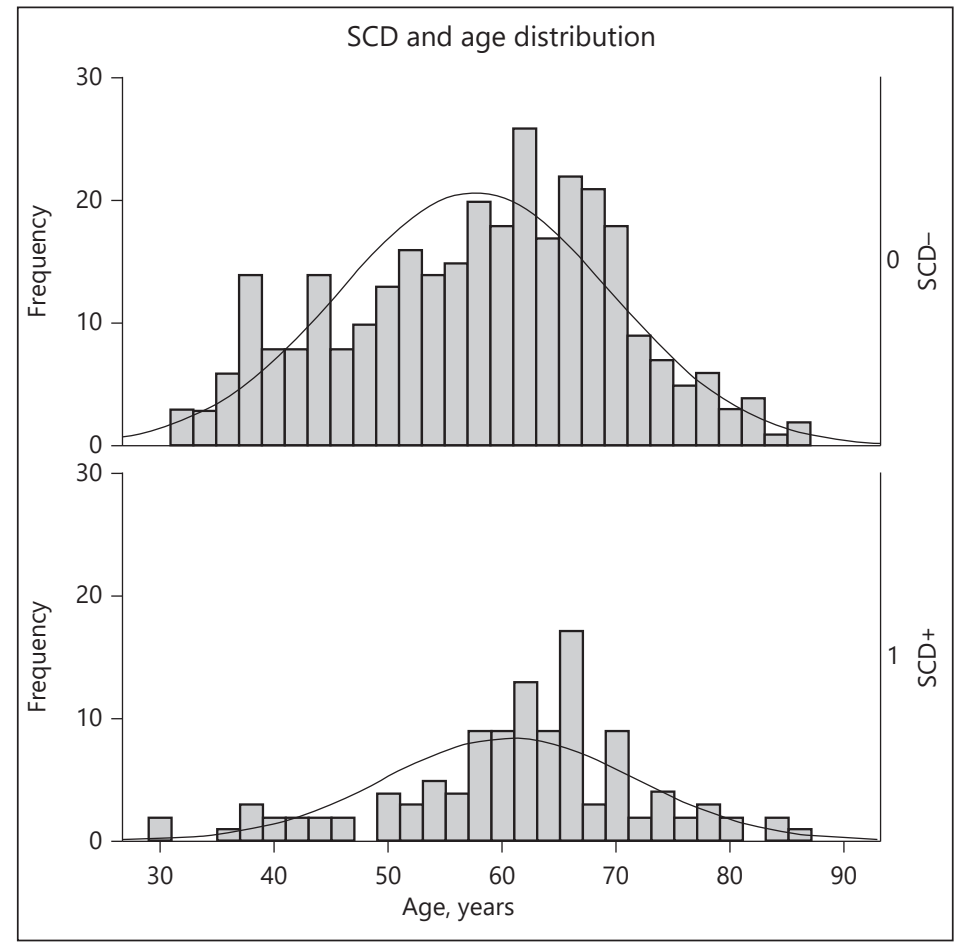

cated that the average heart rate of SCD+_50 members ( $83.3 \pm 10.8$ beats $/ \mathrm{min}$ ) was slightly faster than that of SCD-_50 (80.0 \pm 12.0 beats $/ \mathrm{min})$, with $p<0.05$ (Table 1$)$.

Regarding the AD8 questionnaire, 106 (32.2\%) of those older than 50 years responded "yes" to item 8 (consistent problems with thinking and/or memory). In SCD+_50, 77.0\% of members responded "yes" to this question. Accordingly, consistent problems with thinking and/or memory was the most common cognitive problem faced (Table 2).

This study then explored the relationships between SCD and other physical or mental conditions among participants older than 50 years. The TDQ contained 18 questions with a score from 0 to 3 for low to high frequencies of each question. The TDQ scored from 0 to 54 to indicate a tendency of depression from none to high. Table 2 indicates that groups SCD+_50 and SCD-_50 exhibited significant differences in TDQ scores. According to the obtained result, individuals with depressive tendencies (TDQ 19) were more likely to be assigned to SCD+_50 (15.6\%) than SCD-_50 (2.3\%), with $p<0.001$. Individuals without depressive tendencies (TDQ 8) were more likely to be classified into SCD-_50 (79.4\%) than SCD+_50 (50.5\%), with $p<0.001$.

The PSQI used 19 items to create 7 components of sleep quality. Each component contained a 0-3 interval scale. Summation of the 7 components produced a global score ranging from 0 to 21 to represent overall sleep quality from good to not good. PSQI results indicated that the overall sleep quality of the SCD+_50 group (PSQI_total $=7.2 \pm 4.1$ ) was inferior to that of the SCD-_50 group (PSQI_total $=5.8 \pm 3.7$ ), with $p<0.01$. The percentages of high sleep quality (defined as PSQI_total $\leq 5$, PSQI_good) in SCD+_50 and SCD-_50 were 40.2 and $58.1 \%$, respectively $(p<0.01)$. More detailed analysis of sleep quality revealed that the SCD-_50 group performed more favorably than the SCD+_50 group in terms of subjective sleep quality (PSQI_QUAL; SCD+_50: $1.6 \pm 0.9$; $S C D-\_50: 1.3 \pm 8.2 ; p<0.01$ ), sleep disturbance (PSQI_DIST; SCD+_50: $1.5 \pm 0.6$; SCD-_50: $1.3 \pm 0.5 ; p<0.01$ ), use of sleep medication (PSQI_ 
Table 1. Basic information and laboratory tests of the population aged above 50

\begin{tabular}{|c|c|c|c|c|c|c|}
\hline & $\mathrm{SCD}+$ & SCD- & $p$ value & $\mathrm{SCD}+\_50$ & SCD-_50 & $p$ value \\
\hline Cases, $n$ & $115(27.0)$ & $311(73.0)$ & & $100(30.4)$ & $229(69.6)$ & \\
\hline \multicolumn{7}{|l|}{ Demographics } \\
\hline Age, years & $60.7 \pm 10.9$ & $57.6 \pm 12.0$ & $0.012^{*}$ & $63.8 \pm 7.6$ & $63.3 \pm 7.9$ & 0.571 \\
\hline Sex (male) & $36(31.3)$ & $102(32.8)$ & 0.770 & $31(31.0)$ & 77 (33.6) & 0.641 \\
\hline \multicolumn{7}{|l|}{ Body compositions } \\
\hline SBP, mm Hg & $132.9 \pm 19.3$ & $134.1 \pm 18.5$ & 0.579 & $134.6 \pm 19.0$ & $137.5 \pm 16.7$ & 0.161 \\
\hline $\mathrm{DBP}, \mathrm{mm} \mathrm{Hg}$ & $78.4 \pm 10.6$ & $80.1 \pm 11.6$ & 0.158 & $79.1 \pm 10.4$ & $81.2 \pm 10.5$ & 0.093 \\
\hline HR, beats/min & $83.2 \pm 10.5$ & $80.2 \pm 11.8$ & $0.015^{*}$ & $83.3 \pm 10.8$ & $80.0 \pm 12.0$ & $0.015^{*}$ \\
\hline Height, $\mathrm{cm}$ & $157.3 \pm 8.0$ & $158.5 \pm 8.4$ & 0.193 & $156.7 \pm 7.7$ & $157.4 \pm 8.4$ & 0.472 \\
\hline Weight, kg & $63.3 \pm 12.8$ & $64.0 \pm 12.7$ & 0.636 & $63.0 \pm 11.7$ & $63.3 \pm 12.4$ & 0.826 \\
\hline Waist, $\mathrm{cm}$ & $83.2 \pm 11.4$ & $82.6 \pm 10.3$ & 0.609 & $83.8 \pm 11.0$ & $83.3 \pm 10.0$ & 0.684 \\
\hline BMI & $25.4 \pm 3.9$ & $25.4 \pm 4.2$ & 0.871 & $25.6 \pm 3.7$ & $25.5 \pm 4.1$ & 0.841 \\
\hline \multicolumn{7}{|c|}{ Laboratory data of blood tests } \\
\hline Creatinine, mg/dL & $0.8 \pm 0.3$ & $0.8 \pm 0.2$ & 0.430 & $0.8 \pm 0.3$ & $0.8 \pm 0.2$ & 0.773 \\
\hline eGFR, \% & $87.4 \pm 23.3$ & $90.0 \pm 22.9$ & 0.293 & $85.4 \pm 23.7$ & $85.9 \pm 22.8$ & 0.876 \\
\hline ALT, U/L & $26.0 \pm 15.5$ & $27.2 \pm 18.2$ & 0.516 & $25.8 \pm 14.5$ & $28.0 \pm 18.5$ & 0.285 \\
\hline Sugar AC, mg/dL & $105.1 \pm 35.8$ & $104.5 \pm 35.6$ & 0.883 & $107.2 \pm 37.9$ & $107.6 \pm 34.1$ & 0.925 \\
\hline $\mathrm{HbA}_{1 \mathrm{c}}, \%$ & $6.1 \pm 1.3$ & $6.1 \pm 1.0$ & 0.562 & $6.2 \pm 1.4$ & $6.2 \pm 1.0$ & 0.832 \\
\hline T-chol, mg/dL & $200.6 \pm 38.1$ & $200.4 \pm 40.0$ & 0.952 & $198.3 \pm 38.0$ & $201.8 \pm 40.0$ & 0.455 \\
\hline $\mathrm{LDL}, \mathrm{mg} / \mathrm{dL}$ & $134.0 \pm 36.7$ & $132.8 \pm 37.1$ & 0.759 & $131.5 \pm 36.1$ & $134.1 \pm 38.2$ & 0.561 \\
\hline $\mathrm{HDL}, \mathrm{mg} / \mathrm{dL}$ & $58.9 \pm 17.6$ & $57.8 \pm 17.5$ & 0.564 & $59.2 \pm 17.4$ & $58.1 \pm 17.0$ & 0.592 \\
\hline $\mathrm{TG}, \mathrm{mg} / \mathrm{dL}$ & $127.4 \pm 76.9$ & $135.6 \pm 108.0$ & 0.459 & $126.2 \pm 76.2$ & $135.6 \pm 76.4$ & 0.306 \\
\hline WBC, $n \times 10^{3} / \mathrm{mm}^{3}$ & $6.1 \pm 1.5$ & $6.0 \pm 1.5$ & 0.456 & $6.0 \pm 1.5$ & $5.8 \pm 1.6$ & 0.323 \\
\hline $\mathrm{Hb}, \mathrm{g} / \mathrm{dL}$ & $13.5 \pm 1.5$ & $13.8 \pm 1.5$ & 0.110 & $13.5 \pm 1.5$ & $13.8 \pm 1.4$ & 0.080 \\
\hline НCT, \% & $39.9 \pm 3.6$ & $40.7 \pm 3.8$ & 0.050 & $39.9 \pm 3.6$ & $40.8 \pm 3.7$ & $0.040 *$ \\
\hline $\mathrm{MCV}, \mathrm{fL}$ & $88.5 \pm 6.8$ & $87.4 \pm 7.3$ & 0.166 & $88.6 \pm 6.6$ & $88.1 \pm 6.7$ & 0.513 \\
\hline Platelets, $n \times 10^{3} / \mathrm{mm}^{3}$ & $259.4 \pm 65.0$ & $253.4 \pm 58.4$ & 0.360 & $254.4 \pm 62.7$ & $244.8 \pm 54.9$ & 0.161 \\
\hline
\end{tabular}

$* p<0.05$ as statistically significant. Results are presented as numbers with percentages in parentheses or as means \pm standard deviation. SCD, subjective cognitive decline; SBP, systolic blood pressure; DBP, diastolic blood pressure; HR, heart rate; $\mathrm{BMI}$, body mass index; eGFR, estimated glomerular filtration rate; $\mathrm{ALT}$, alanine aminotransferase; $\mathrm{AC}$, ante cibum; $\mathrm{HbA}_{1 \mathrm{c}}$, glycated hemoglobin; T-chol, total cholesterol; LDL, low-density cholesterol; HDL, high-density cholesterol; TG, triglyceride; WBC, white blood cell; Hb, hemoglobin; HCT, hematocrit; MCV, mean corpuscular volume.

MED; SCD+_50: $0.8 \pm 1.3$; SCD-_50: $0.4 \pm 0.9 ; p<0.01$ ), and daytime dysfunction (PSQI_DYS; $\mathrm{SCD}+50: 0.5 \pm 0.7$; $\mathrm{SCD}-\_50: 0.3 \pm 0.6 ; p<0.05$ ).

Thirty-six items of the SF-36 produced 8 scaled scores, each of which ranged from 0 to 100. The higher the score, the less disability. The statistically significant results of SF-36 assessment revealed that the $\mathrm{SCD}+50$ group exhibited an overall quality of life lower than that of the SCD-_50 group, receiving relatively low scores in all 8 dimensions: physical functioning (SF-36_PF; $p<0.001$ ), physical role functioning (SF-36_RP; $p<0.001$ ), bodily pain (SF-36_BP; $p=0.001$ ), general health (SF-36_GH; $p<0.001$ ), vitality (SF-36_VT; $p<0.001$ ), social role functioning (SF-36_SF; $p<0.001$ ), emotional role functioning (SF-36_RE; $p<0.001$ ), and mental health (SF-36_MH; $p<0.001$ ).

The HPLP-S contained 6 domains, 4 items in each domain, and a scaled score from 1 (low intensity) to 4 (high intensity) of each item. The statistically significant results of the HPLP-S indicate that the SCD+_50 group had less optimal lifestyles for health promotion than the SCD-_50 group regarding lifestyles of nutrition (HPLP-S_N; SCD+_50: 7.2 \pm 2.8 ; SCD-_50: 8.0 $\pm 2.5 ; p<0.05$ ), self-actualization (HPLP-S_SA; SCD+_50: $6.1 \pm 3.6$; SCD-_50: $7.7 \pm 3.1 ; p<$ 0.001), and stress management (HPLP-S_SM; SCD+_50: 5.9 \pm 3.2 ; SCD-_50:6.6 $\pm 2.9 ; p<0.05$ ). 
Table 2. Subjective cognitive complaints and health questionnaires of patients aged above 50 years

\begin{tabular}{|c|c|c|c|}
\hline & $\mathrm{SCD}+50$ & SCD-_50 & $p$ value \\
\hline Cases, $n$ & $100(30.4)$ & $229(69.6)$ & \\
\hline \multicolumn{4}{|l|}{ Subjective cognitive decline } \\
\hline Valid AD8 questionnaire & $100(100)$ & $229(100)$ & \\
\hline AD8_total & $3.1 \pm 1.5$ & $0.3 \pm 0.5$ & $<0.001^{*}$ \\
\hline AD8_1_judgment & $30(30.0)$ & $4(1.7)$ & $<0.001^{*}$ \\
\hline AD8_2_interest & $41(41.0)$ & $3(1.3)$ & $<0.001^{*}$ \\
\hline AD8_3_repeat & $48(48.0)$ & $12(5.2)$ & $<0.001^{*}$ \\
\hline AD8_4_learn & $30(30.0)$ & $9(3.9)$ & $<0.001^{*}$ \\
\hline AD8_5_date & $36(36.0)$ & $7(3.1)$ & $<0.001^{*}$ \\
\hline AD8_6_finance & $17(17.0)$ & $1(0.4)$ & $<0.001^{*}$ \\
\hline AD8_7_appointment & $32(32.0)$ & $4(1.7)$ & $<0.001^{*}$ \\
\hline AD8_8_memory & $77(77.0)$ & $29(12.7)$ & $<0.001^{*}$ \\
\hline \multicolumn{4}{|l|}{ Depression tendency } \\
\hline Valid TDQ questionnaire & $90(90)$ & $219(91.7)$ & \\
\hline TDQ_total & $10.6 \pm 8.7$ & $4.6 \pm 5.4$ & $<0.001^{*}$ \\
\hline Depression (TDQ $\geq 19$ ) & $14(15.6)$ & $5(2.3)$ & $<0.001^{*}$ \\
\hline No depressive tendency (TDQ $\leq 8)$ & $45(50.0)$ & $174(79.5)$ & $<0.001^{*}$ \\
\hline \multicolumn{4}{|l|}{ Sleep quality } \\
\hline Valid PSQI questionnaire & 97 (97) & $227(99.1)$ & \\
\hline PSQI_total & $7.2 \pm 4.1$ & $5.8 \pm 3.7$ & $0.004^{*}$ \\
\hline PSQI_good (PSQI_total $\leq 5$ ) & $39(40.2)$ & $132(58.1)$ & $0.003^{*}$ \\
\hline PSQI_QUAL & $1.6 \pm 0.9$ & $1.3 \pm 8.2$ & $0.009 *$ \\
\hline PSQI_LAT & $1.5 \pm 1.2$ & $1.2 \pm 1.0$ & 0.059 \\
\hline PSQI_DUR & $0.8 \pm 1.0$ & $0.8 \pm 0.9$ & 0.914 \\
\hline PSQI_EFF & $0.6 \pm 1.0$ & $0.6 \pm 1.0$ & 0.540 \\
\hline PSQI_DIST & $1.5 \pm 0.6$ & $1.3 \pm 0.5$ & $0.004^{*}$ \\
\hline PSQI_MED & $0.8 \pm 1.3$ & $0.4 \pm 0.9$ & $0.003^{*}$ \\
\hline PSQI_DYS & $0.5 \pm 0.7$ & $0.3 \pm 0.6$ & $0.043^{*}$ \\
\hline \multicolumn{4}{|l|}{ Quality of life } \\
\hline Valid SF-36 questionnaire & 99 (99) & $228(99.6)$ & \\
\hline SF-36_PF & $77.3 \pm 23.1$ & $87.3 \pm 15.1$ & $<0.001^{*}$ \\
\hline SF-36_RP & $66.3 \pm 42.6$ & $87.1 \pm 31.2$ & $<0.001^{*}$ \\
\hline SF-36_BP & $74.0 \pm 24.3$ & $81.8 \pm 19.0$ & $0.001^{*}$ \\
\hline SF-36_GH & $54.2 \pm 24.2$ & $64.5 \pm 19.3$ & $<0.001^{*}$ \\
\hline SF-36_VT & $60.8 \pm 23.7$ & $73.6 \pm 17.5$ & $<0.001^{*}$ \\
\hline SF-36_SF & $83.0 \pm 19.6$ & $93.9 \pm 11.3$ & $<0.001^{*}$ \\
\hline SF-36_RE & $72.5 \pm 41.1$ & $90.9 \pm 25.8$ & $<0.001^{*}$ \\
\hline SF-36_MH & $64.3 \pm 21.7$ & $79.5 \pm 22.2$ & $<0.001^{*}$ \\
\hline \multicolumn{4}{|l|}{ Health promotion lifestyle } \\
\hline Valid HPLP-S questionnaire & $100(100)$ & $229(100)$ & \\
\hline HPLP-S_E & $4.6 \pm 3.2$ & $5.2 \pm 2.8$ & 0.099 \\
\hline HPLP-S_N & $7.2 \pm 2.8$ & $8.0 \pm 2.5$ & $0.012^{*}$ \\
\hline HPLP-S_SA & $6.1 \pm 3.6$ & $7.7 \pm 3.1$ & $<0.001^{*}$ \\
\hline HPLP-S_IS & $7.0 \pm 3.6$ & $7.6 \pm 2.8$ & 0.138 \\
\hline HPLP-S_HR & $5.4 \pm 3.6$ & $5.2 \pm 3.3$ & 0.593 \\
\hline HPLP-S_SM & $5.9 \pm 3.2$ & $6.6 \pm 2.9$ & $0.039 *$ \\
\hline
\end{tabular}

${ }^{*} p<0.05$ as statistically significant. Results are presented as numbers with percentages in parentheses, scores as means \pm standard deviation. AD8, dementia screening interview; TDQ, Taiwanese Depression Questionnaire; PSQI, Pittsburgh Sleep Quality Index; PSQI_QUAL, overall subjective sleep quality; PSQI_LAT, sleep latency; PSQI_DUR, sleep duration; PSQI_EFF, sleep efficiency; PSQI_DIST, sleep disturbance; PSQI_ MED, need medication to sleep; PSQI_DYS, dysfunction due to sleepiness; SF-36, the Short-Form (36-item) Health Survey; SF-36_PF, physical functioning score; SF-36_RP, physical role functioning score; SF-36_BP, body pain score; SF-36_GH, general health score; SF-36_VT, vitality score; SF-36_SF, social role functioning score; SF-36_RE, emotional role functioning score; SF-36_MH, mental health score; HPLP-S, short form of the Chinese Health Promoting Lifestyle Profile; HPLP-S_E, exercise score; HPLP-S_N, nutrition score; HPLP-S_SA, self-actualization score; HPLP-S_IS, interpersonal support score; HPLP-S_HR, health response score; HPLP-S_ SM, stress management score. 
Fig. 4. Multiple-domain involvements of SCD. Increased age increased the likelihood of SCD. Meanwhile, people with SCD tend to have a less satisfactory sleep quality, less optimal quality of life, higher tendency of depression, and less positive health-promoting lifestyle than people without SCD.

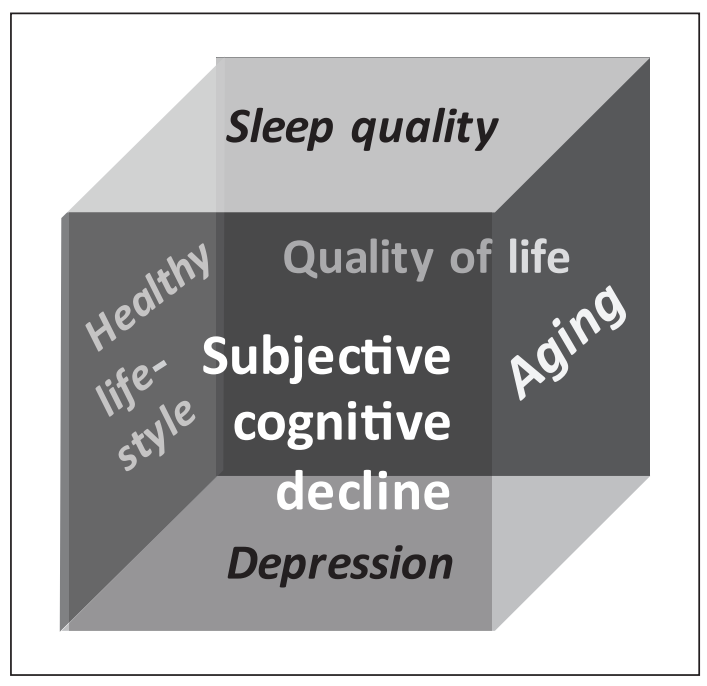

\section{Discussion}

A total of 426 valid AD8 questionnaires were collected. Participants who received a score higher than 2 in this screening scale were deemed to have SCD, whereas those with a score of 0-1 were without SCD. Old age was the most significant difference between the SCD+ and SCD- groups. After participants younger than 50 years had been excluded, multifaceted assessment scales were adopted, and the results indicated that the SCD-_50 group lived with more health-seeking life styles, had more satisfactory quality of life, reported a better quality of sleep, and had less tendency of depression than the SCD+_50 group. In summary, individuals with SCD exhibit not only suboptimal performance in subjective cognition, but also in numerous dimensions other than cognitive abilities.

Research limitations are detailed as follows. Because this community-based study employed a cross-sectional approach, individuals may have had diverse motivations for participating in this program. This study had a potential selection bias and was unable to achieve random sampling comparable to censuses. Consequently, our interpretation for subjective cognitive decline in community dwellers may also be biased. Furthermore, potential interrater and intrarater biases were considered. We have made efforts to reduce these biases by regularly training testers to standardize the interviews of participants.

Self-perceived memory difficulty is the commonest subjective cognitive problem in older adults. A 20-year study monitored community-dwelling women aged 65 years and older with perceived memory difficulty and confirmed that subjective memory impairment in an early stage is indeed a warning sign of neurodegenerative disease [17]. After the manifestation of SCD, the patient may gradually develop impaired cognitive function and exhibit deteriorated work performance [18]; in addition, the patients are prone to develop psychiatric or mental comorbidities. Zlatar et al. [19] studied the relationship between SCD and depression, and revealed that compared with similar individuals without SCD, those with SCD developed comorbidities with depression at a higher rate. Systematic literature review has also revealed the interaction between depression and SCD [7], indicating that depression may be an early symptom of dementia [20] or comorbidity with early cognitive impairment.

Sleep disorders, daytime sleepiness, and forgetfulness are three descriptions commonly reported together by patients. Chronic sleep disorder leads to poor subjective and objective 
cognitive performance [21]. Community research on older adults discovered that people with poor sleep quality are prone to develop cognitive impairment in the long term [22]. The present study indicated that individuals with SCD exhibit poor sleep quality and low overall quality of life, which are consistent with the conclusions of a recent meta-analysis stating that individuals with severe SCD are prone to have a relatively low quality of life [23]. Furthermore, overall physical and mental health is closely related to a healthpromoting lifestyle. Studies have revealed that a highly health-promoting lifestyle is associated with a lower likelihood of depressive tendencies [24] and engenders a higher quality of life [25]. Additionally, a comprehensive review of depression and quality of life clearly demonstrated that older adults with depressive tendencies have a relatively low overall quality of life [26]. Based on literature review and our study, SCD, depression, sleep quality, quality of life, and health-promoting lifestyle are all correlated and affect lives holistically (Fig. 4).

In this cross-sectional study, a relatively rapid resting heart rate was also a difference between individuals with SCD and those without, regardless of age. Although a large-scale study in Denmark failed to demonstrate a significant correlation between scores of objective cognitive tests and resting heart rate [27], resting heart rate is closely associated with overall health. People with faster resting heart rates are more likely to develop asymptomatic inflammation [28] and have a higher cardiovascular risk [29]. A 16-year panel study in Copenhagen, Denmark, discovered that people with rapid resting heart rate have relatively poor physical fitness. An increase in 10 beats/min in resting heart rate was associated with a $16 \%$ greater likelihood of death [30]. Heart rate is controlled by the sinoatrial node of the right atrium. The sinoatrial node is controlled by the autonomic nervous system; stimulated sympathetic nerves accelerate heart rate, whereas stimulated parasympathetic nerves decelerate heart rate [31]. In this study, participants with SCD had a faster heart rate on average, implying greater cardiovascular risk, poorer physical fitness, or potential cardiac dysautonomia. However, this inference requires further assessment, such as an analysis of heart rate variability [32], of cardiovascular function, physical fitness, and the autonomic nervous system to confirm the correlations.

Community medical cohort research is one of the most effective means to understand cognitive impairment, particularly the relationship between incidence and environment, which cannot be observed in case-controlled or hospital-based studies. For example, the Framingham Heart Study, in Massachusetts, USA, was a cohort study beginning in 1948 with 5,209 participants. A total of 5,124 people were included in the second generation of this study in 1971, and 4,095 people were involved in the third generation in 2005. The Framingham Heart Study reported the incidence of subjective and objective cognitive impairment over 3 generations from the past 50 years, revealing that the proportion of $\varepsilon 4$ allele of the apolipoprotein E gene has not changed over generations, whereas significant variations could be observed in stricter control of blood pressure, more prevalent use of hypolipidemic agents, higher educational attainment, and lowered smoking prevalence. Furthermore, the overall prevalence of cognitive problems decreased over generations, and the decline in vascular dementia was statistically significant [33]. The Framingham cohort study revealed that longterm monitoring of a community enables researchers to determine the effects of generation changes and the macroeconomic environment on cognition [34]. In the spirit of service and research, the Community Medicine Research Center of Keelung Chang Gung Memorial Hospital has been conducting long-term monitoring of the cognitive health of residents of the northern coastal communities of Taiwan, providing them with holistic care for their mental and physical health to improve their quality of life. 


\section{Acknowledgment}

We are grateful to the Community Medicine Research Center and Department of Medical Research and Development of Keelung Chang Gung Memorial Hospital for supporting this study. This study was funded by Chang Gung Research Project No. CLRPG2H0041 and No. CRRPG2G0071. We also like to thank Mrs. Shu-Hua Chen for assisting data collection and study maintenance.

\section{Statement of Ethics}

All the subjects had been well informed about the study and had given their consents before entering the study.

\section{Disclosure Statement}

All of the authors declared that there's no existing and possible conflict of interest.

\section{References}

1 World Health Organization: Dementia fact sheet No. 362, April 2016. Geneva: World Health Organization; 2016

2 Langa KM, Levine DA. The diagnosis and management of mild cognitive impairment: a clinical review. JAMA. 2014 Dec;312(23):2551-61.

3 Petersen RC. Clinical practice. Mild cognitive impairment. N Engl J Med. 2011 Jun;364(23):2227-34.

4 Juncos-Rabadan O, Pereiro AX, Facal D, Rodriguez N, Lojo C, Caamaño JA, et al. Prevalence and correlates of cognitive impairment in adults with subjective memory complaints in primary care centres. Dement Geriatr Cogn Disord. 2012;33(4):226-32.

5 Jessen F, Amariglio RE, van Boxtel M, Breteler M, Ceccaldi M, Chételat G, et al.; Subjective Cognitive Decline Initiative (SCD-I) Working Group. A conceptual framework for research on subjective cognitive decline in preclinical Alzheimer's disease. Alzheimers Dement. 2014 Nov;10(6):844-52.

6 Rabin LA, Smart CM, Amariglio RE. Subjective Cognitive Decline in Preclinical Alzheimer's Disease. Annu Rev Clin Psychol. 2017 May;13(1):369-96.

7 Hill NL, Mogle J, Wion R, Munoz E, DePasquale N, Yevchak AM, et al. Subjective Cognitive Impairment and Affective Symptoms: A Systematic Review. Gerontologist. 2016 Dec;56(6):e109-27.

8 Galvin JE, Roe CM, Powlishta KK, Coats MA, Muich SJ, Grant E, et al. The AD8: a brief informant interview to detect dementia. Neurology. 2005 Aug;65(4):559-64.

9 Galvin JE, Roe CM, Coats MA, Morris JC. Patient's rating of cognitive ability: using the AD8, a brief informant interview, as a self-rating tool to detect dementia. Arch Neurol. 2007 May;64(5):725-30.

10 Yang YH, Galvin JE, Morris JC, Lai CL, Chou MC, Liu CK. Application of AD8 questionnaire to screen very mild dementia in Taiwanese. Am J Alzheimers Dis Other Demen. 2011 Mar;26(2):134-8.

11 Lee Y, Yang MJ, Lai TJ, Chiu NM, Chau TT. Development of the Taiwanese Depression Questionnaire. Chang Gung Med J. 2000 Nov;23(11):688-94.

12 Buysse DJ, Reynolds CF 3rd, Monk TH, Berman SR, Kupfer DJ. The Pittsburgh Sleep Quality Index: a new instrument for psychiatric practice and research. Psychiatry Res. 1989 May;28(2):193-213.

13 Tsai PS, Wang SY, Wang MY, Su CT, Yang TT, Huang CJ, et al. Psychometric evaluation of the Chinese version of the Pittsburgh Sleep Quality Index (CPSQI) in primary insomnia and control subjects. Qual Life Res. 2005 Oct;14(8):1943-52.

14 Tseng HM, Lu JF, Tsai YJ. Assessment of health-related quality of life in Taiwan. II. Norming and validation of SF-36 Taiwan version. Taiwan J Public Health. 2003;22:512-8.

15 Lu JF, Tseng HM, Tsai YJ. Assessment of health-related quality of life in Taiwan. I. Development and psychometric testing of SF-36 Taiwan version. Taiwan J Public Health. 2003;22:501-11.

16 Wei MH, Lu CM. Development of the Short-Form Chinese Health Promoting Lifestyle Profile. J Health Educ. 2005;24:25-46.

17 Kaup AR, Nettiksimmons J, LeBlanc ES, Yaffe K. Memory complaints and risk of cognitive impairment after nearly 2 decades among older women. Neurology. 2015 Nov;85(21):1852-8. 
18 Koppara A, Wagner M, Lange C, Ernst A, Wiese B, König HH, et al. Cognitive performance before and after the onset of subjective cognitive decline in old age. Alzheimers Dement (Amst). 2015 May;1(2):194-205.

19 Zlatar ZZ, Muniz MC, Espinoza SG, Gratianne R, Gollan TH, Galasko D, et al. Subjective Cognitive Decline, Objective Cognition, and Depression in Older Hispanics Screened for Memory Impairment. J Alzheimers Dis. 2018;63(3):949-56.

20 Wilson RS, Barnes LL, Mendes de Leon CF, Aggarwal NT, Schneider JS, Bach J, et al. Depressive symptoms, cognitive decline, and risk of AD in older persons. Neurology. 2002 Aug;59(3):364-70.

21 Bastien CH, Fortier-Brochu E, Rioux I, LeBlanc M, Daley M, Morin CM. Cognitive performance and sleep quality in the elderly suffering from chronic insomnia. Relationship between objective and subjective measures. J Psychosom Res. 2003 Jan;54(1):39-49.

22 Blackwell T, Yaffe K, Laffan A, Ancoli-Israel S, Redline S, Ensrud KE, et al.; Osteoporotic Fractures in Men (MrOS) Study Group. Associations of objectively and subjectively measured sleep quality with subsequent cognitive decline in older community-dwelling men: the MrOS sleep study. Sleep (Basel). 2014 Apr;37(4): 655-63.

23 Hill NL, McDermott C, Mogle J, Munoz E, DePasquale N, Wion R, et al. Subjective cognitive impairment and quality of life: a systematic review. Int Psychogeriatr. 2017 Dec;29(12):1965-77.

24 Hua Y, Wang B, Wallen GR, Shao P, Ni C, Hua Q. Health-promoting lifestyles and depression in urban elderly Chinese. PLoS One. 2015 Mar;10(3):e0117998.

25 Rakhshani T, Shojaiezadeh D, Lankarani KB, Rakhshani F, Kaveh MH, Zare N. The association of healthpromoting lifestyle with quality of life among the Iranian elderly. Iran Red Crescent Med J. 2014 Sep; 16(9):e18404.

26 Sivertsen H, Bjørkløf GH, Engedal K, Selbæk G, Helvik AS. Depression and quality of life in older persons: a review. Dement Geriatr Cogn Disord. 2015 Sep;40:311-339.

27 Wod M, Jensen MT, Galatius S, Hjelmborg JB, Jensen GB, Christensen K. Resting Heart Rate Is Not Associated with Cognitive Function. Neuroepidemiology. 2018;50(3-4):160-7.

28 Sajadieh A, Nielsen OW, Rasmussen V, Hein HO, Abedini S, Hansen JF. Increased heart rate and reduced heartrate variability are associated with subclinical inflammation in middle-aged and elderly subjects with no apparent heart disease. Eur Heart J. 2004 Mar;25(5):363-70.

29 Fox K, Borer JS, Camm AJ, Danchin N, Ferrari R, Lopez Sendon JL, et al.; Heart Rate Working Group. Resting heart rate in cardiovascular disease. J Am Coll Cardiol. 2007 Aug;50(9):823-30.

30 Jensen MT, Suadicani P, Hein HO, Gyntelberg F. Elevated resting heart rate, physical fitness and all-cause mortality: a 16-year follow-up in the Copenhagen Male Study. Heart. 2013 Jun;99(12):882-7.

31 Gordan R, Gwathmey JK, Xie LH. Autonomic and endocrine control of cardiovascular function. World J Cardiol. 2015 Apr;7(4):204-14.

32 Billman GE. Heart rate variability: a historical perspective. Front Physiol. 2011 Nov; 2:86.

33 Zhang J. Mapping neuroinflammation in frontotemporal dementia with molecular PET imaging. J Neuroinflammation. 2015 May;12(1):108.

34 Satizabal CL, Beiser AS, Chouraki V, Chêne G, Dufouil C, Seshadri S. Incidence of Dementia over Three Decades in the Framingham Heart Study. N Engl J Med. 2016 Feb;374(6):523-32. 